\title{
Statistical mechanics of sum frequency generation spectroscopy for the liquid-vapor interface of dilute aqueous salt solutions
}

\author{
Joyce Noah-Vanhoucke, ${ }^{1}$ Jared D. Smith, ${ }^{2}$ and Phillip L. Geissler ${ }^{1,2}$ \\ ${ }^{1}$ Department of Chemistry, University of California, Berkeley, California 94720 \\ ${ }^{2}$ Chemical Sciences Division Lawrence Berkeley National Lab, Berkeley, California 94720
}

(Dated: January 2, 2009)

\begin{abstract}
We demonstrate a theoretical description of vibrational sum frequency generation (SFG) at the boundary of aqueous electrolyte solutions. This approach identifies and exploits a simple relationship between SFG lineshapes and the statistics of molecular orientation and electric field. Our computer simulations indicate that orientational averages governing SFG susceptibility do not manifest ion-specific shifts in local electric field, but instead, ion-induced polarization of subsurface layers. Counterbalancing effects are obtained for monovalent anions and cations at the same depth. Ions held at different depths induce an imbalanced polarization, suggesting that ion-specific effects can arise from weak, long ranged influence on solvent organization.
\end{abstract}




\section{INTRODUCTION}

Interest in ion solvation at interfaces has been renewed by recent developments in nonlinear spectroscopic experiments (notably sum frequency generation and second harmonic generation), as well as computer simulation studies that point to unexpected aqueous surface phenomena for simple inorganic anions. Specifically, these simulations suggest that large atomic anions, such as iodide, experience an effective attraction for the liquid-vapor interface[1-5], counter to predictions from cornerstone theories of ion solvation in bulk water[6, 7]. Although a growing number of experimental studies support this surprising notion of surface enhancement[8-11], detailed inferences from the data rest on methods that remain controversial[12].

Sum frequency generation $(\mathrm{SFG})$ is the only second-order optical process that provides information on the vibrational response of molecules at an interface. Detailed theoretical formulations permit calculation of SFG intensity, e.g., for liquid-vapor interfaces, from computer simulations [13-17], but do not by themselves offer a transparent physical picture of its relationship to intermolecular structure. We have recently presented a convenient, albeit approximate, approach to such calculations for the specific case of hydroxyl stretching at aqueous liquid-vapor interfaces. By neglecting solvent dynamics and casting the influence of a proton's surroundings as a Stark effect, it reduces SFG susceptibility to a superposition of simple orientational averages resolved by fluctuations in electric field [18]. These approximations, which underlie very accurate theories of Raman spectra for bulk aqueous solutions [19, 20], clarify the spectroscopic consequences of molecular alignment and distortions of intermolecular arrangements near the liquid surface. In particular, they highlight the weakness of orientational bias for interfacial molecules that donate hydrogen bonds [18].

In this work, we apply such a computational approach to the liquid-vapor interface of salt water. Specifically, we consider the influence of dissolved halide anions and sodium cations on hydroxyl stretching of dilute HOD solutes in $\mathrm{D}_{2} \mathrm{O}$ solvent. Section II reviews the orientational averages that govern resonant SFG susceptibility within our approximations. Our numerical methods are described in detail in Sec. III, and results of molecular dynamics simulations are presented Sec. IV. These calculations identify those aspects of interfacial ion solvation that strongly determine spectroscopic observables. They suggest that ion-specific effects may, in fact, be closely related to spontaneous charge layering at the interface. 
The polarizability of larger atomic anions, such as iodide, has been assigned a central role in the disputed phenomenon of aqueous surface adsorption[3-5]. For ease of interpretation, we focus attention in this paper on results for model ions that lack polarizability, but are held at fixed depth. In doing so, we anticipate that the direct spectroscopic influence of induced solute dipoles need not be as significant as is their impact on the ions' spatial distribution. In Sec. IV B we present results for polarizable ion models that confirm this expectation. These calculations were performed by an exact reweighting of data obtained from nonpolarizable models, as we will describe in detail.

We conclude in Sec. V with a discussion of the implications of our results for interpreting SFG spectra of electrolyte liquid-vapor interfaces.

\section{ORIENTATIONAL AVERAGES RESPONSIBLE FOR SFG}

SFG response is proportional to the squared magnitude of the second-order susceptibility $\boldsymbol{\chi}$, which in turn is proportional to the average molecular hyperpolarizability, $\boldsymbol{\chi} \propto\langle\boldsymbol{\beta}\rangle$. The theory developed in Ref. 18 emphasizes the importance of orientational averages of the form $\left\langle j_{\alpha} k_{\gamma} l_{\zeta}\right\rangle_{\mathcal{E}}$ in determining the absorptive part of SFG susceptibility. Here, $j_{\alpha}, k_{\gamma}$, and $l_{\zeta}$ are

projections of the Cartesian unit vectors $\hat{\mathbf{j}}, \hat{\mathbf{k}}$, and $\hat{\mathbf{l}}$ defining a water molecule's reference frame onto the $\alpha, \gamma$, and $\zeta$ axes of the laboratory frame. Averages $\langle\cdot\rangle_{\mathcal{E}}$ for an HOD molecule are conditioned on the solution's electric field $\mathcal{E}$ evaluated at the proton's position and projected onto the $\mathrm{OH}$ bond. Within our approximations, variations in hydroxyl stretching frequency are directly proportional to this fluctuating electrostatic coordinate, $\omega=\omega^{(0)}+Q \mathcal{E}$, where $Q$ and $\omega^{(0)}$ are positive constants[21, 22].

For the most widely used combination of input light polarizations, ssp, our estimate for the imaginary part of the resonant hyperpolarizability is given by

$$
\operatorname{Im} \boldsymbol{\beta}_{x x z}^{(\mathrm{R})}(\omega)=\frac{\pi}{Q} P(\mathcal{E}) \sum_{j, k, l} \alpha_{j k}^{\prime} \mu_{l}^{\prime}\left\langle j_{x} k_{x} l_{z}\right\rangle_{\mathcal{E}}
$$

where the laboratory $z$-axis points outward from and normal to the liquid's surface. The superposition of orientational averages in Eq. 1 is weighted by the probability distribution over the entire system, $P(\mathcal{E})$, which is overwhelmingly dominated by contributions from the slab's bulk-like interior. The frequency-independent coefficients $\alpha_{j k}^{\prime} \mu_{l}^{\prime}$ are determined by matrix elements of the dipole and polarizability derivatives with respect to the vibrational 


\begin{tabular}{|c|c|}
\hline$z$ & $\begin{array}{l}\text { Cartesian coordinate in the direction normal to the liquid-vapor interface, } \\
\text { relative to the liquid's center of mass }\end{array}$ \\
\hline$z_{\text {ion }}$ & position of an ionic solute in the $z$-direction \\
\hline$z_{\text {Gibbs }}$ & position of the Gibbs dividing surface, i.e., half of the thickness of the liquid slab \\
\hline$\Delta z_{\text {ion }}$ & position of an ionic solute relative to the Gibbs dividing surface, i.e., $z_{\text {ion }}-z_{\text {Gibbs }}$ \\
\hline $\mathcal{E}$ & $\begin{array}{l}\text { instantaneous electric field generated by the solution, evaluated at the } \\
\text { proton's position and projected onto the corresonding hydroxyl bond }\end{array}$ \\
\hline$P(\mathcal{E})$ & probability distribution of $\mathcal{E}$ at thermal equilibrium \\
\hline $\mathbf{u}, \mathbf{u}^{\prime}$ & $\begin{array}{l}\text { unit vectors in the HOD molecular reference frame, pointing along the } \mathrm{OH} \text { and } \\
\text { OD bond directions, respectively }\end{array}$ \\
\hline$u_{z}, u_{z}^{\prime}$ & $\begin{array}{l}\text { projections of the unit vectors } \mathbf{u} \text { and } \mathbf{u}^{\prime} \\
\text { onto the outward surface normal of the liquid slab }\end{array}$ \\
\hline$\left\langle u_{z}\right\rangle_{\mathcal{E}, z_{\mathrm{ion}}},\left\langle u_{z}^{\prime}\right\rangle_{\mathcal{E}, z_{\mathrm{ion}}}$ & $\begin{array}{l}\text { conditional averages of } u_{z} \text { and } u_{z}^{\prime} \text { for hydroxyl groups with a given value of } \mathcal{E} \text {, } \\
\text { when an ion is located at height } z_{\text {ion }}\end{array}$ \\
\hline$\xi_{\text {ion }}$ & $\begin{array}{l}\text { instantaneous electrostatic force per unit charge exerted by the solution } \\
\text { on an ionic solute, in the } z \text {-direction }\end{array}$ \\
\hline
\end{tabular}

TABLE I: Table of important variables used throughout the paper.

coordinate. See Ref. 18 for a detailbed account of this expression's origin and for precise definitions of the constant parameters involved.

The conditional averages $\left\langle j_{x} k_{x} l_{z}\right\rangle_{\mathcal{E}}$ quantify the correlation between a molecule's hydrogen bonding environment, as characterized by $\mathcal{E}$, and its orientational bias due to the interface. Of the 18 distinct choices of the set $\{j, k, l\}$, eight are zero due to symmetry. Of the remaining ten choices, four are negligible due to the likely very small values of $\alpha_{j k}^{\prime}$ and $\mu_{l}^{\prime}$ that multiply them. The final six are significantly redundant in their frequency dependence. They can each be written, to a good approximation, as a linear combination of the simpler averages $\left\langle u_{z}\right\rangle_{\mathcal{E}}$ and $\left\langle u_{z}^{\prime}\right\rangle_{\mathcal{E}}$, where $\hat{\mathbf{u}}$ and $\hat{\mathbf{u}}^{\prime}$ denote unit vectors along the $\mathrm{OH}$ and $\mathrm{OD}$ bonds, respectively, of an HOD molecule.

The essence of an SFG lineshape in our approach thus reduces to two functions of electric field (or, equivalently, frequency), each multiplied by the electric field distribution $P(\mathcal{E})$. 
The first, $\left\langle u_{z}\right\rangle_{\mathcal{E}}$, quantifies the orientation of $\mathrm{OH}$ bonds whose protons experience a given electric field $\mathcal{E}$. The second, and slightly more subtle, $\left\langle u_{z}^{\prime}\right\rangle_{\mathcal{E}}$, quantifies the orientation of OD bonds whose corresponding protons experience a particular value of $\mathcal{E}$. The effect of dissolved ions on SFG follows from changes in these two functions. The distribution $P(\mathcal{E})$ of electric field fluctuations over the entire slab is extremely insensitive to their presence and location at the ionic strengths we consider. Our simulations consider different ranges of ion depth separately. Because these ranges are narrow, we effectively compute orientational averages $\left\langle u_{z}\right\rangle_{\mathcal{E}, z_{\text {ion }}}$ and $\left\langle u_{z}^{\prime}\right\rangle_{\mathcal{E}, z_{\text {ion }}}$ conditioned not only on electric field, but also on the ion's vertical coordinate, $z_{\text {ion }}$.

\section{SIMULATION METHODS}

We have investigated the $\mathrm{SFG}$ response of dilute $\mathrm{HOD}$ in $\mathrm{D}_{2} \mathrm{O}$ for a series of electrolyte solutions consisting of either one or two ions dissolved in water. In particular, we simulated the dynamics of $N=512$ molecules interacting via the $\mathrm{SPC} / \mathrm{E}$ potential [23] computed with Ewald sums, at ambient temperature and at fixed densities that enforce liquid-vapor coexistence. The system consists of a slab of liquid with two air-liquid interfaces. The slab dimensions are $20 \AA \times 20 \AA \times 40 \AA$ with nearly $60 \AA$ of vapor in the $z$-direction. The box dimensions are $L_{x} \times L_{y} \times L_{z}=20 \AA \times 20 \AA \times 100 \AA$. Statistics were accumulated for the positive, $z>0$, half of the slab system, where the $z$-coordinate of the hydrogen atom was used to determine to which half of the slab a particular $\mathrm{OH}$ group belongs. The procedure for creating this interfacial system, the implications of our symmetry breaking convention, and further aspects of our simulation methodology are described in greater detail in Ref. 18 . Interactions between ions and water and between different ions were computed according to models developed by Dang and coworkers [24] (absent contributions due to molecular and ionic polarizability). Our simulations explored only $\mathrm{Na}^{+}$as the cation species, and several halide anions: $\mathrm{F}^{-}, \mathrm{Cl}^{-}, \mathrm{Br}^{-}$, and $\mathrm{I}^{-}$. Single-ion systems are, in principle, unstable due to interactions between periodic images that are not electroneutral. By neglecting the zerowavevector contribution in Ewald sums, we effectively stabilize these systems by introducing a uniform neutralizing background charge density of opposite sign.

Molecular dynamics simulations were performed using the LAMMPS software package [25]. The SHAKE algorithm [26] was used to maintain rigid molecular geometries in these 
calculations. Equilibration runs employed a Nosé-Hoover thermostat [27] for at least 200 ps, while production runs were propagated by integrating Newton's equations of motion using the velocity Verlet algorithm [28] for at least $2 \mathrm{~ns}$. In both cases $\Delta t=1 \mathrm{fs}$ served as the fundamental time step of integration.

Ions, when present without a partner counterion, were allowed to explore limited ranges of depth $\Delta z_{\text {ion }}=z_{\text {ion }}-z_{\text {Gibbs }}$, where $z_{\text {Gibbs }}$ is the value of $z$ at which the solvent density falls to half its bulk value. This biasing of the ion position was achieved via an external potential,

$$
U_{\text {bias }}=u_{0}\left(z_{\text {ion }}-\bar{z}-z_{0}\right)^{4}
$$

where

$$
\bar{z}=\frac{\sum_{i=1}^{N} z_{i}^{(\mathrm{O})} h\left(z_{i}^{(\mathrm{O})}\right)}{\sum_{i=1}^{N} h\left(z_{i}^{(\mathrm{O})}\right)}
$$

is an effective center of mass for the slab. The counting function $h(z)=(1+\exp [a(z-$ $\left.\left.\left.L_{z} / 2\right)\right]\right)^{-1} \times\left(1+\exp \left[-a\left(z+L_{z} / 2\right)\right]\right)^{-1}$, with $a=4 \AA^{-1}$, smoothly restricts the center of mass calculation to the central simulation cell. The summations run over all molecules in the system, and the superscripts $(\mathrm{O})$ indicate that a molecule's position is determined by its oxygen atom's location. By choosing $u_{0}=0.5 \mathrm{kcal} / \mathrm{mol}^{4}$ we were able to restrict an ion's motion in the $z$-direction to within $\pm 1 \AA$ of $z_{0}$. Simulations were performed for a series of ion depths: $z_{0}=0,9 \AA, 11 \AA, 13 \AA, 15 \AA, 17 \AA, 19 \AA$, and $21 \AA$ (corresponding roughly to $\Delta z_{\text {ion }} \approx-20 \AA,-9 \AA,-7 \AA,-5 \AA,-3 \AA,-1 \AA$, and $\left.1 \AA\right)$. For simulations including a dissolved ion pair, the bias (2) was applied to the anion, while the cation's position was restricted with an additional bias potential,

$$
U_{\text {cation }}(\mathbf{r}, z)=c\left(z_{s}-\left(z_{\text {anion }}-z_{\text {cation }}\right)\right)^{2}+d\left(r_{0}-|\mathbf{r}|\right)^{2}
$$

where $z_{s}$ is the desired separation of the cation and anion in the $z$-direction, $\mathbf{r}$ is the vector pointing from the cation to the anion projected onto the $x y$-plane, and $r_{0}$ is the desired sepration in the $x y$-plane. For the choice of parameters $c=d=2 \mathrm{kcal} / \mathrm{mol} \AA^{2}, z_{s}=3.5 \AA$ and $r_{0}=6 \AA$, the ions reside in reasonable proximity, both situated near the interface, but separated in the $z$-direction. Specifically, the anion is confined to $\Delta z_{\text {anion }} \approx-1 \AA \pm 1 \AA$, while the cation explores a range of depth $\Delta z_{\text {cation }} \approx-5 \AA \pm 2 \AA$. 


\section{SIMULATION RESULTS}

\section{A. Dilute Electrolyte Solutions}

Single-ion simulations with periodic boundary conditions are in a sense unphysical, since it is not possible in the laboratory to study solutions bearing a macroscopic excess of positive or negative charge. Computer simulations of such systems can nonetheless provide a useful way to understand separately the effects of an anion or cation on its surroundings, and on spectroscopic observables. We also report on electroneutral simulations that include one anion and one cation.

Inspired by simulations and experiments that explore a series of halide ions at the airwater interface, we have investigated $\mathrm{F}^{-}, \mathrm{Cl}^{-}, \mathrm{Br}^{-}$, and $\mathrm{I}^{-}$independently as the anionic species. In our simulation models, which lack polarizability, these ions differ essentially only by size. Figure 1 shows the functions $\left\langle u_{z}\right\rangle_{\mathcal{E}, z_{\text {ion }}}$ and $\left\langle u_{z}^{\prime}\right\rangle_{\mathcal{E}, z_{\text {ion }}}$ for each of these ions compared to results for a neat water-air system. In all cases, the ion was restrained to a narrow range of depth, $z_{\text {ion }} \approx z_{\text {Gibbs }}-1 \AA$. The most notable aspects of these results are, first, that no substantial new spectroscopic features appear due to ion solvation and, second, that the weak changes we do detect are nearly identical across the halide series. In sharp contrast, halide ion solvation in bulk produces marked Raman shifts, once ascribed to "structure making" or "structure breaking" activity of the ions, but now understood as the consequence of strong electric fields exerted by the anion on adjacent hydroxyl groups [21]. Because those anionic fields decay rapidly over short distances, small ions such as $\mathrm{F}^{-}$generate distinctly different shifts than do larger ions such as $\mathrm{I}^{-}$. Such short-ranged, ion-specific modifications are not apparent in the orientational averages we have calculated.

Despite the differing spectroscopic effects of halide ions in the bulk and at the interface, the local solvation structure of each ion is only weakly dependent on depth, within the non-polarizable models we use, for ion positions that occur with appreciable probability $[29,30]$. We have confirmed this fact by examining, for example, the average number of water molecules in each ion's first solvation shell for $z_{\text {ion }} \leq z_{\text {Gibbs }}+4 \AA$. More pertinently, electric field distributions for protons adjacent to the ion in this depth range are nearly identical to those computed for bulk solutions. The ion-specific frequency shifts that these $\mathrm{OH}$ groups experience do not contribute noticeably to computed SFG simply as a consequence of sym- 
metry. Local solvation structure is largely determined by the very strong hydrogen bonds donated by solvent molecules to the anion. For the models we simulate, this local structure does not change substantially in the vicinity of the Gibbs dividing surface [29]. Significant changes do not emerge in the case of iodide solutes until the ion is constrained to reside well above the interface, $z_{\text {ion }} \geq z_{\text {Gibbs }}+4 \AA$. Fluoride anions retain bulk-like first solvation shells even farther into the vapor phase, investigated in our studies up to $z_{\text {ion }} \approx z_{\text {Gibbs }}+15 \AA$. Because the arrangement of solvating molecules in bulk is, on average, symmetric about the

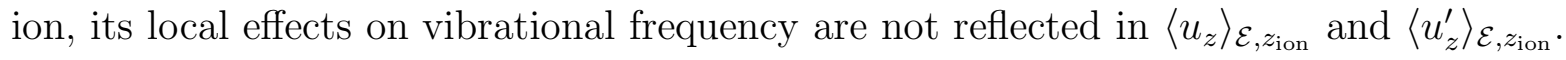

Though small, the ion-independent changes in orientational averages we have observed can be spectroscopically significant if they occur near the peak of $P(\mathcal{E})$. Both $\left\langle u_{z}\right\rangle_{\mathcal{E}, z_{\text {ion }}}$ and $\left\langle u_{z}^{\prime}\right\rangle_{\mathcal{E}, z_{\text {ion }}}$ do, in fact, show systematic positive shifts throughout the range $\mathcal{E}<-1 \mathrm{~V} / \AA$. Since these shifts do not arise from directly solvating molecules, they must be longer-ranged in character. We indeed find that ions induce a weak but long-ranged orientational bias on water molecules that extends many molecular diameters into the slab. This polarization of the subsurface layers has little to do with the ion's size or local solvation structure, arising instead from a weak imbalance in electrostatic forces over the nm scale. The weakness of this effect is evidenced by the nearly frequency-independent offset of $\left\langle u_{z}^{\prime}\right\rangle_{\mathcal{E}, z_{\text {ion }}}$ due to the ion. Molecular reorientation is so subtle as to be insensitive to its immediate hydrogen bonding environment.

The positive offset of $\left\langle u_{z}\right\rangle_{\mathcal{E}, z_{\text {ion }}}$ due to weak polarization in the range $\mathcal{E}<-1 \mathrm{~V} / \AA$ is accompanied by noticeable frequency dependence. (This function effectively vanishes in neat solvent over the range of frequencies corresponding to hydrogen bond-donating $\mathrm{OH}$ groups.) We attribute this additional effect to slight contractions and expansions of the hydrogen bond network radial to the ion: An $\mathrm{OH}$ group whose proton is directed toward the ion will experience a force from the ion in that direction (which, for most of the subsurface layer, has a positive projection onto the surface normal). With high probability, its hydrogen bonding partner will reside in roughly the same direction, so that motion toward the ion effects shortening of the hydrogen bond and, as a result, shifting of $\mathcal{E}$ to more negative values. An $\mathrm{OH}$ group pointing away from the ion, on the other hand, will tend to retract from its acceptor, generating positive frequency shifts. Computed correlations between $r_{\mathrm{OH}}$, the distance between a proton and its hydrogen bond acceptor, and $\mathcal{E}$ support this picture[18].

Our calculations indicate that the influence of anion solvation, as reflected in orientational 
averages relevant to SFG, extends over lengths appreciably larger than a molecular diameter. On these scales, the charge asymmetry of a water molecule, which is crucial to hydrogen bonding, no longer dominates solvent response to electrostatic forces. It may therefore not be surprising that the anionic effects discussed above are recapitulated in opposite senses for a single cation at the interface. Figure 2 shows calculations of $\left\langle u_{z}\right\rangle_{\mathcal{E}, z_{\text {ion }}}$ and $\left\langle u_{z}^{\prime}\right\rangle_{\mathcal{E}, z_{\text {ion }}}$ from simulations including $\mathrm{Na}^{+}$(and no anion) at the interface. Systematic negative shifts over the range $\mathcal{E}<\mathcal{E}^{*}$, as well as an upward slope, of $\left\langle u_{z}\right\rangle_{\mathcal{E}, z_{\text {ion }}}$, can be rationalized using ideas analogous to those we have presented in the context of halide anions.

Any physically realizable electrolyte solution must of course possess both positive and negative ions. At extremely low ionic strength, we could think of such a solution as a concatenation of the single-ion systems we have simulated. We could therefore approximate the spectroscopic effects of a dissolved ion pair by averaging results obtained for isolated counterions held at the same depth. Figure 3 shows the results of such a simple-minded average. The individual influences of anion and cation are not only comparable in a qualitative sense; they offset one another quantitatively. This cancellation underscores the long-ranged nature of ionic perturbations manifested in the orientational averages we are considering. Within our approximations, a well-separated pair of ions at the same depth produces no change in SFG susceptibility.

Recent computer simulations have raised the unexpected possibility that simple dissolved ion pairs can establish well defined layers at the air-water interface[3, 4]. The effective adsorption of larger halide anions appears to be sensitive to details of intermolecular interactions, and in particular, to ion polarizability, whose effect on SFG we scrutinize closely in Sec. IV B. Here we first ask a question that circumvents this sensitivity: With our approximate treatment of SFG and our non-polarizable interaction model, what are the spectroscopic implications of the vertical charge separation predicted by more sophisticated models? Put more simply, do the effects of anion and cation balance fail to cancel when they reside at different depths below the interface?

We have repeated simulations of the single- $\mathrm{Na}^{+}$system with the cation restrained to a series of depth intervals. Figure 4 shows that the ion-induced orientational changes vanish in a smooth and simple way as the ion moves farther into the bulk. Molecular layers above the ion are generally oriented opposite those below the ion, weakening the net polarization until the neat solvent response is restored. If we repeat the naive averaging procedure to 
calculate SFG susceptibility for a vertically segregated ion pair, it is clear that the balance of orienting effects will be disturbed, as Fig. 5 confirms. The result is a net change in the functions $\left\langle u_{z}\right\rangle_{\mathcal{E}, z_{\text {ion }}}$ and $\left\langle u_{z}^{\prime}\right\rangle_{\mathcal{E} \text {,ion }}$ that, when multiplied by $P(\mathcal{E})$, is considerable. Notably, these modifications to the determinants of second-order susceptibility are similar in form and in magnitude to results obtained from more detailed simulations, as well as from experiment.

We have tested our ideas regarding the opposing influences of an ion pair by performing simulations that include both $\mathrm{Na}^{+}$and $\mathrm{I}^{-}$. Here we probe an extreme that complements our treatment of infinite dilution, in which the two ions are held close together in a configuration chosen to mimic the density profiles of high concentration electrolyte solutions where ion layering is evident. Figure 6 demonstrates that our result for the dilute limit provides a surprisingly accurate estimate for the case of paired ions.

\section{B. Effect of Ion Polarizability on SFG}

Omitting anion polarizability is a potentially serious shortcoming of the molecular model used in the preceding sections to examine SFG response at electrolyte-vapor interfaces. Using computer simulations, Tobias and coworkers have shown that the attraction for the interface of larger halide anions such as $\mathrm{I}^{-}$is substantially weakened when their dipole fluctuations are neglected[4]. The solvation effects underlying strong charge layering (which does not occur in our non-polarizable model) could also introduce pronounced asymmetry in an ion's first coordination shell[31]. In that case, the longer-ranged contributions we have described would be supplemented, or perhaps even outweighed, by orientation and frequency shifts of hydroxyl groups adjacent to the ion.

We have examined the possible importance of induced solute dipoles by computing the

orientational averages $\left\langle u_{z}\right\rangle_{\mathcal{E}, z_{\text {ion }}}$ and $\left\langle u_{z}^{\prime}\right\rangle_{\mathcal{E}, z_{\text {ion }}}$ for a polarizable ion held fixed at a series of depths below the interface, i.e., we have determined the effects of polarizability on the conditional averages $\left\langle u_{z}\right\rangle_{\mathcal{E}, z_{\text {ion }}}$ and $\left\langle u_{z}^{\prime}\right\rangle_{\mathcal{E}, z_{\text {ion }}}$. From these quantities, SFG susceptibility can be calculated using

$$
\operatorname{Im} \boldsymbol{\beta}_{x x z}^{(\mathrm{R})}(\omega)=\frac{\pi}{Q} \int d z_{\text {ion }} p\left(z_{\text {ion }}\right) P\left(\mathcal{E} \mid z_{\text {ion }}\right) \sum_{j, k, l} \alpha_{j k}^{\prime} \mu_{l}^{\prime}\left\langle j_{x} k_{x} l_{z}\right\rangle_{\mathcal{E}, z_{\text {ion }}}
$$

At the ion concentrations of our simulations, the dependence of $P\left(\mathcal{E} \mid z_{\text {ion }}\right)$ on ion depth is undetectable. We can thus safely write the hyperpolarizability as a linear combination 
of spatially averaged functions $\int d z_{\text {ion }} p\left(z_{\text {ion }}\right)\left\langle j_{x} k_{x} l_{z}\right\rangle_{\mathcal{E}, z_{\text {ion }}}$, and subsequently in terms of the simpler quantities $\int d z_{\text {ion }} p\left(z_{\text {ion }}\right)\left\langle u_{z}\right\rangle_{\mathcal{E}, z_{\text {ion }}}$ and $\int d z_{\text {ion }} p\left(z_{\text {ion }}\right)\left\langle u_{z}^{\prime}\right\rangle_{\mathcal{E}, z_{\text {ion }}}$. This simplification accomplishes a separation of two distinct effects of ion polarizability. The first, which has been widely discussed, is a spatial redistribution of ion depths described by changes in $p\left(z_{\text {ion }}\right)$. Here we exclusively consider the second effect, namely, changes, due to polarizability, in the orientational averages $\left\langle u_{z}\right\rangle_{\mathcal{E}, z_{\text {ion }}}$ and $\left\langle u_{z}^{\prime}\right\rangle_{\mathcal{E}, z_{\text {ion }}}$ when ion depth is restrained.

To assess such consequences of ion polarizability, we introduce the solute's dipole $\boldsymbol{\mu}$ as a dynamical variable, and supplement the potential energy studied in previous sections, $U^{(0)}$, with terms regulating fluctuations in $\boldsymbol{\mu}$ :

$$
U^{(\mathrm{pol})}=U^{(0)}-\boldsymbol{\xi}_{\text {ion }} \cdot \boldsymbol{\mu}+\frac{|\boldsymbol{\mu}|^{2}}{2 \alpha} .
$$

Here, $\alpha$ is the ion polarizability, and vector $\boldsymbol{\xi}_{\text {ion }}$ is the instantaneous electric field exerted by the solution on the ion. Note that $\boldsymbol{\xi}_{\text {ion }}$ is a function of all particle positions.

We compute averages over the Boltzmann distribution defined by Eq. 6 using its nonpolarizable counterpart as a reference system. Integrating out fluctuations in $\boldsymbol{\mu}$, we obtain for an arbitrary observable quantity $A$

$$
\langle A\rangle^{(\mathrm{pol})}=\frac{\left\langle A \exp \left(\beta \alpha \xi^{2} / 2\right)\right\rangle^{(0)}}{\left\langle\exp \left(\beta \alpha \xi^{2} / 2\right)\right\rangle^{(0)}},
$$

where $\xi \equiv\left|\boldsymbol{\xi}_{\text {ion }}\right|$. Superscripts "(pol)" and "(0)" denote averages over fluctuations in the polarizable and non-polarizable systems, respectively. For one of the orientational averages relevant to SFG, we have specifically

$$
\left\langle u_{z}\right\rangle_{\mathcal{E}, z_{\text {ion }}}^{(\mathrm{pol})}=\frac{\int d \xi \exp \left(\beta \alpha \xi^{2} / 2\right) P^{(0)}\left(\mathcal{E}, z_{\text {ion }}, \xi\right)\left\langle u_{z}\right\rangle_{\mathcal{E}, z_{\text {ion }}, \xi}^{(0)}}{\int d \xi \exp \left(\beta \alpha \xi^{2} / 2\right) P^{(0)}\left(\mathcal{E}, z_{\text {ion }}, \xi\right)}
$$

(Results for the other key orientational average $\left\langle u_{z}^{\prime}\right\rangle_{\mathcal{E}, z_{\text {ion }}}^{(\text {pol }}$ yield conclusions analogous to those drawn below.) We have defined $P^{(0)}\left(\mathcal{E}, z_{\text {ion }}, \xi\right)$ as the joint probability distribution for equilibrium fluctuations of $\xi$ (the magnitude of the solution's electric field acting on the ion), $\mathcal{E}$, and $z_{\text {ion }}$ in the non-polarizable ensemble.

Fig. 7 shows simulation results for $\left\langle u_{z}\right\rangle_{\mathcal{E}, z_{\text {ion }}, \xi}^{(0)}$ as a function of $\mathcal{E}$, for an iodide anion held at $z_{\text {ion }}=19 \AA \pm 1 \AA$ (using the bias potentials discussed in Sec. III) and for values of $\xi$ spanning the range of typical equilibrium fluctuations. The profound insensitivity to $\xi$ evident in these data, especially in the hydrogen bonding region of $\mathcal{E}$, are representative of simulations we have performed for a variety of ion depths and identities. This fact, together 
with Eq. 8, suggests that $\left\langle u_{z}\right\rangle_{\mathcal{E}, z_{\text {ion }}}^{(\text {pol })}=\left\langle u_{z}\right\rangle_{\mathcal{E}, z_{\text {ion }}}^{(0)}$ to an excellent approximation. Carrying out the integrations in Eq. 8 numerically, we indeed find negligible differences between these quantities, as shown in Fig. 8.

For the molecular models under consideration, the influence of ion polarizability lies almost entirely in modifying the spatial distributions $p\left(z_{\text {ion }}\right)$. The statistics of electric field fluctuations that modulate $\mathrm{OH}$ stretching frequency are nearly identical in polarizable and non-polarizable models when the ion depth is restrained. These results provide strong justification for the approach we have adopted in previous sections, and for the conclusion that ion-specific aspects of SFG have little to do with local solvation structure.

\section{CONCLUSIONS}

We have shown that ion-specific effects on SFG spectra in sodium halide solutions can have very different origins than those observed in bulk Raman spectra. Frequency shifts for $\mathrm{OH}$ groups proximal to the ion, which dominate changes in bulk spectra[21], have little influence on SFG response due to the nearly symmetric organization, on average, of an anion's nearby solvation structure. For the molecular model we have studied, the interface has little influence on these local solvent geometries. When placed at the same depths, all halide ions we have studied affect relevant orientational averages in precisely the same way, reflecting weak, long-ranged polarization of subsurface layers. Since cations induce an exactly opposing response, these effects would not be noticeable on macroscopic scales in a neutral solution whose cation and anion density profiles are identical. This conclusion is robust to the inclusion of dipole induction on polarizable anions.

Noticeable spectroscopic influence of dissolved salts might instead arise from the specific way charged solutes are distributed in space relative to the interface. We have shown that computed SFG response is, in fact, sensitive to the kind of charge layering suggested by previous simulation studies for larger halide anions. As a result, spectroscopic ion specificity at the interface could well reflect variations in surface density profiles across the halide series. 


\section{Acknowledgments}

This work was supported by the Director, Office of Science, Office of Basic Energy Sciences, Materials Sciences and Engineering Division, of the U.S. Department of Energy under Contract No. DE-AC02-05CH11231. The authors also thank Rich Saykally for useful discussions.

[1] D. Liu, G. Ma, L. Levering, and H. Allen, J. Phys. Chem. B 108, 2252 (2004).

[2] P. Petersen and R. Saykally, Chem. Phys. Lett. 397, 51 (2004).

[3] P. Jungwirth and D. J. Tobias, J. Phys. Chem. B 105, 10468 (2001).

[4] P. Jungwirth and D. J. Tobias, J. Phys. Chem. B 106, 6361 (2002).

[5] L. Vrbka, M. Mucha, B. Minofar, P. Jungwirth, E. Brown, and D. Tobias, Curr. Op. Colloid Interface Sci. 9, 67 (2004).

[6] L. Onsager and N. Samaras, J. Chem. Phys. 2, 528 (1934).

[7] A. A. Rashin and B. Honig, J. Phys. Chem. 89, 5588 (1985).

[8] J. Cheng, C. Vecitis, M. Hoffmann, and A. Colussi, J. Phys. Chem. B (Letter) 110, 25598 (2006).

[9] L. M. Pegram and J. M. Thomas Record, J. Phys. Chem. B 111, 5411 (2007).

[10] S. Ghosal, J. C. Hemminger, H. Bluhm, B. S. Mun, E. L. Hebenstreit, G. Ketteler, D. F. Ogletree, F. G. Requejo, and M. Salmeron, Science 307, 563 (2005).

[11] P. B. Petersen and R. J. Saykally, Annu. Rev. Chem. 57, 333 (2006).

[12] E. A. Raymond and G. L. Richmond, J. Phys. Chem. B 108, 5051 (2004).

[13] A. Morita and J. T. Hynes, Chem. Phys. 258, 371 (2000).

[14] A. Morita and J. T. Hynes, J. Phys. Chem. B 106, 673 (2002).

[15] J. L. Skinner (2008), personal communication.

[16] E. C. Brown, M. Mucha, P. Jungwirth, and D. J. Tobias, J. Phys. Chem. B 109, 7934 (2005).

[17] V. Buch, T. Tarbuck, G. L. Richmond, H. Groenzin, I. Li, and M. J. Shultz, J. Chem. Phys. 127, 204710 (2007).

[18] J. Noah-Vanhoucke, J. D. Smith, and P. L. Geissler, J. Phys. Chem. B (2008), submitted.

[19] J. D. Smith, C. D. Cappa, K. R. Wilson, R. C. Cohen, P. L. Geissler, and R. J. Saykally, 
Proc. Nat. Acad. Sci. USA 102, 14171 (2005).

[20] P. L. Geissler, J. Am. Chem. Soc. 127, 14930 (2005).

[21] J. D. Smith, R. J. Saykally, and P. L. Geissler, J. Am. Chem. Soc. 129, 13847 (2007).

[22] J. D. Eaves, A. Tokmakoff, and P. L. Geissler, J. Phys. Chem. A 109, 9424 (2005).

[23] H. J. Berendsen, J. R. Grigera, and T. P. Straatsma, J. Phys. Chem. 91, 6269 (1987).

[24] L. X. Dang, J. Phys. Chem. B 106, 10388 (2002).

[25] S. Plimpton, J. Comp. Phys. 117, 1 (1995), http://lammps.sandia.gov.

[26] J.-P. Ryckaert, G. Ciccotti, and H. J. Berendsen, J. Comput. Phys. 23, 327 (1977).

[27] w. G. Hoover, Phys. Rev. A 31, 1695 (1985).

[28] W. C. Swope and H. C. Andersen, J. Chem. Phys. 76, 637 (1982).

[29] I. Benjamin, J. Chem. Phys. 95, 3698 (1991).

[30] M. A. Wilson and A. Pohorille, J. Chem. Phys. 95, 6005 (1991).

[31] P. Jungwirth and D. J. Tobias, Chem. Rev. 106, 1259 (2006).

\section{FIGURES}




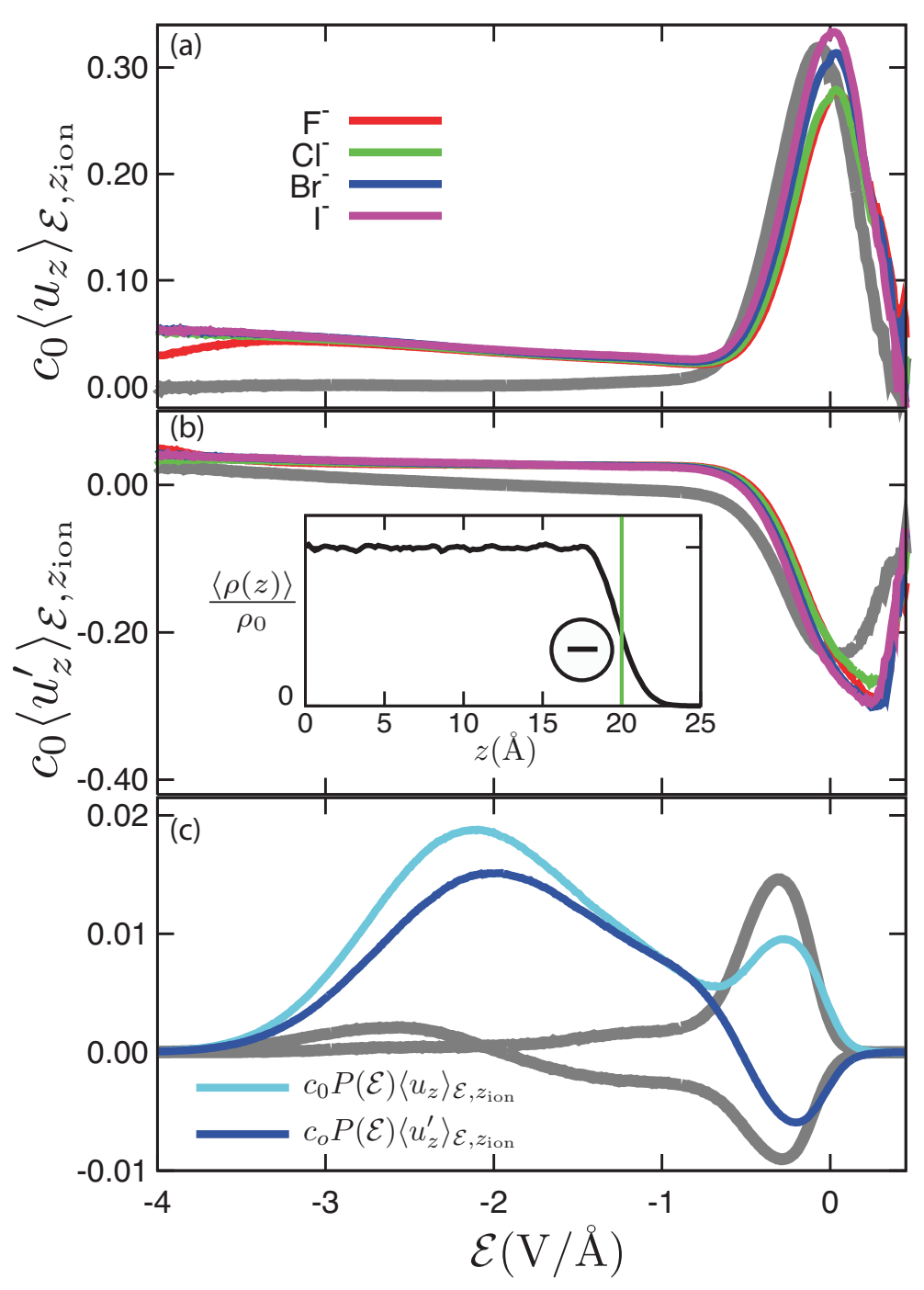

FIG. 1: Average projections of $\mathrm{OH}$ and $\mathrm{OD}$ bond vectors onto the surface normal for systems including a single anion (and no cation) in the liquid slab. Results for $\left\langle u_{z}\right\rangle_{\mathcal{E}}$ and $\left\langle u_{z}^{\prime}\right\rangle_{\mathcal{E}}$ for the neat liquid interface are reproduced here (gray curves) for comparison. All orientational averages are multiplied by a factor $c_{0} \equiv N /\left(2 L_{x} L_{y}\right) \AA^{-2}$ to remove a trivial dependence on system size. Colored lines in (a) and (b) show $c_{0}\left\langle u_{z}\right\rangle_{\mathcal{E}, z_{\text {ion }}}$ and $c_{0}\left\langle u_{z}^{\prime}\right\rangle_{\mathcal{E}, z_{\text {ion }}}$ for four halide ions: $\mathrm{F}^{-}$(red), $\mathrm{Cl}^{-}$ (green), $\mathrm{Br}^{-}$(blue), and $\mathrm{I}^{-}$(violet). In (c) we plot $c_{0} P(\mathcal{E})\left\langle u_{z}\right\rangle_{\mathcal{E}, z_{\text {ion }}}$ and $c_{0} P(\mathcal{E})\left\langle u_{z}^{\prime}\right\rangle_{\mathcal{E}, z_{\text {ion }}}$ for the $\mathrm{Br}^{-}$ system and $c_{0} P(\mathcal{E})\left\langle u_{z}\right\rangle_{\mathcal{E}}$ and $c_{0} P(\mathcal{E})\left\langle u_{z}^{\prime}\right\rangle_{\mathcal{E}}$ for the neat liquid (gray). Other anions yield averages indistinguishable from the case of $\mathrm{Br}^{-}$. In all of these calculations, the ion was restrained to reside close to the interface, with $\left\langle z_{\text {ion }}\right\rangle-z_{\text {Gibbs }}=-1.4 \AA$ and $\sqrt{\left\langle\left(z_{\text {ion }}-\left\langle z_{\text {ion }}\right\rangle\right)^{2}\right\rangle}=0.5 \AA$. The inset in (b) depicts the ion's mean position in relation to the liquid density profile. The Gibbs dividing surface is indicated by the green line. 


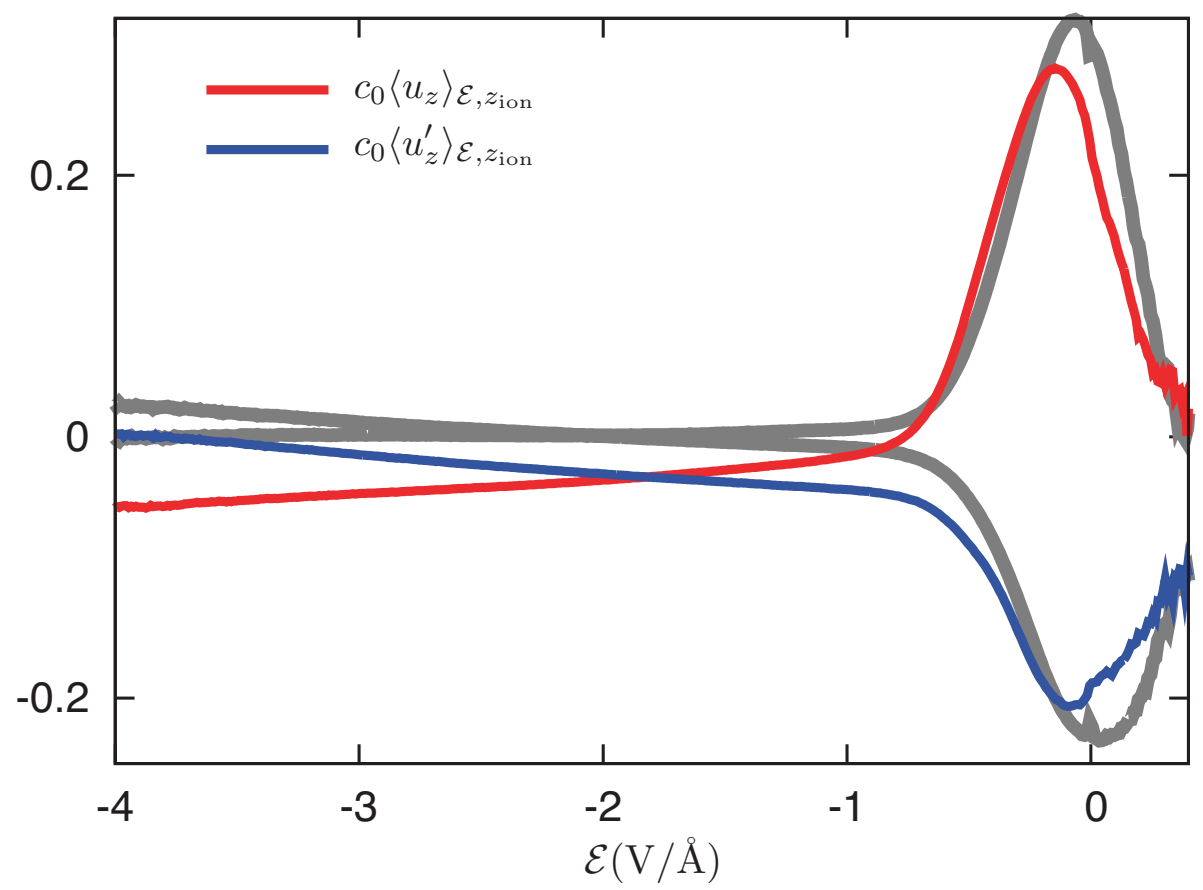

FIG. 2: Average projections of $\mathrm{OH}$ and OD bond vectors onto the surface normal for a liquid slab containing a single $\mathrm{Na}^{+}$ion (and no anion). As in Fig. 1 and in subsequent figures, the factor $c_{0} \equiv N /\left(2 L_{x} L_{y}\right) \AA^{-2}$ renders the calculated averages as intensive quantities. Results for the neat liquid interface are reproduced here (gray curves) for comparison. Colored lines show $c_{0}\left\langle u_{z}\right\rangle_{\mathcal{E}, z_{\text {ion }}}$ (red) and $c_{0}\left\langle u_{z}^{\prime}\right\rangle_{\mathcal{E}, z_{\text {ion }}}$ (blue) as functions of electric field. As for the anion systems reported in Fig. $1, \mathrm{Na}^{+}$was restrained to reside close to the interface, with $\left\langle z_{\text {cation }}\right\rangle-z_{\text {Gibbs }}=-1.3 \AA$ and $\sqrt{\left\langle\left(z_{\text {cation }}-\left\langle z_{\text {cation }}\right\rangle\right)^{2}\right\rangle}=0.5 \AA$. 


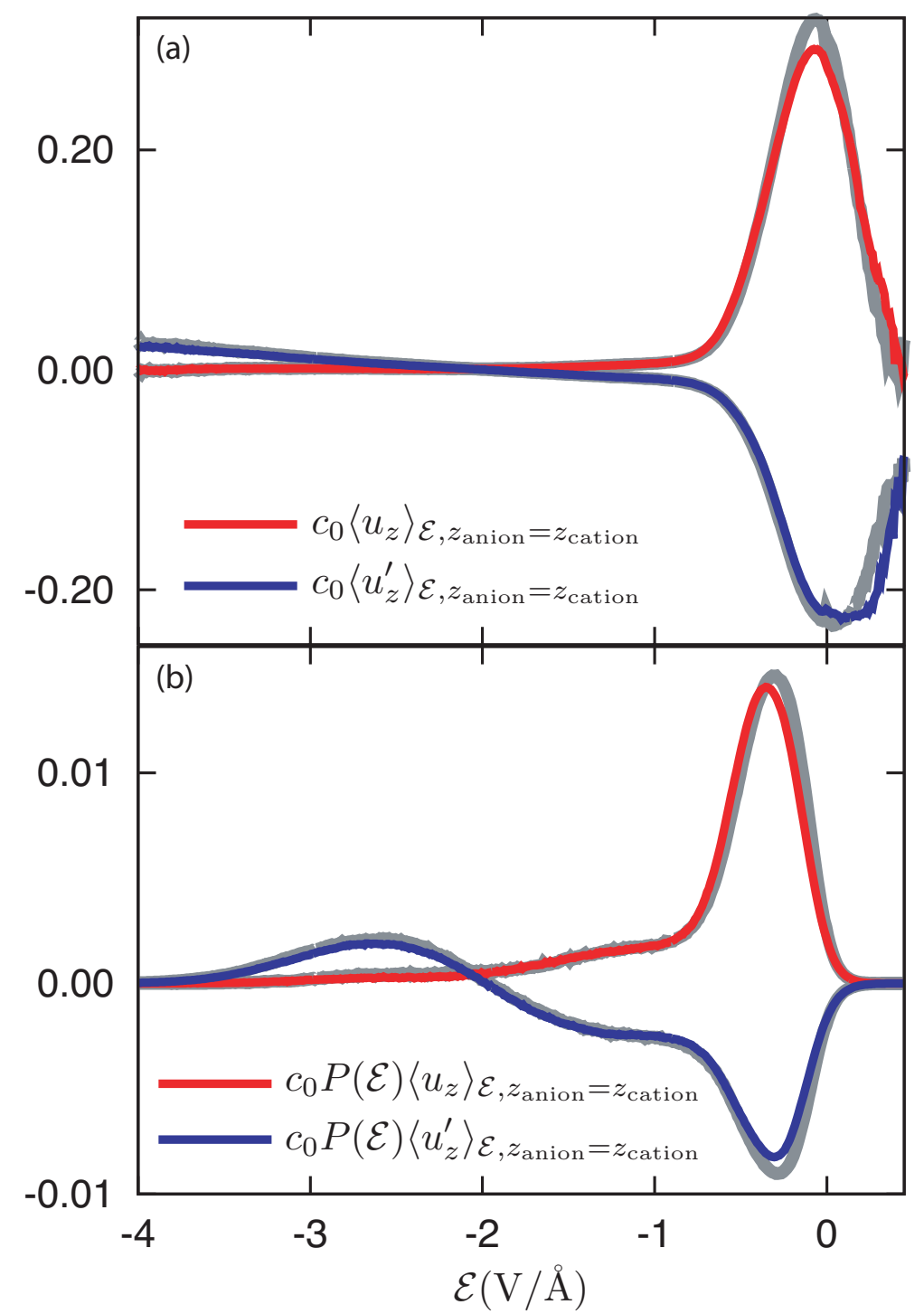

FIG. 3: Estimate of orientational averages governing SFG response for an ion pair, held at identical depths, at infinite dilution. The plotted data were obtained by averaging calculations for a single dissolved $\mathrm{I}^{-}$anion with those for a single dissolved $\mathrm{Na}^{+}$cation, both held at the same depth (as described in the captions of Figs. 1 and 2). Panel (a) shows $c_{0}\left\langle u_{z}\right\rangle_{\mathcal{E}, z_{\text {ion }}}$ and $c_{0}\left\langle u_{z}^{\prime}\right\rangle_{\mathcal{E}, z_{\text {ion }}}$. In (b) these average projections have been weighted by the electric field distribution in order to highlight close similarity with results for the neat liquid (gray). 


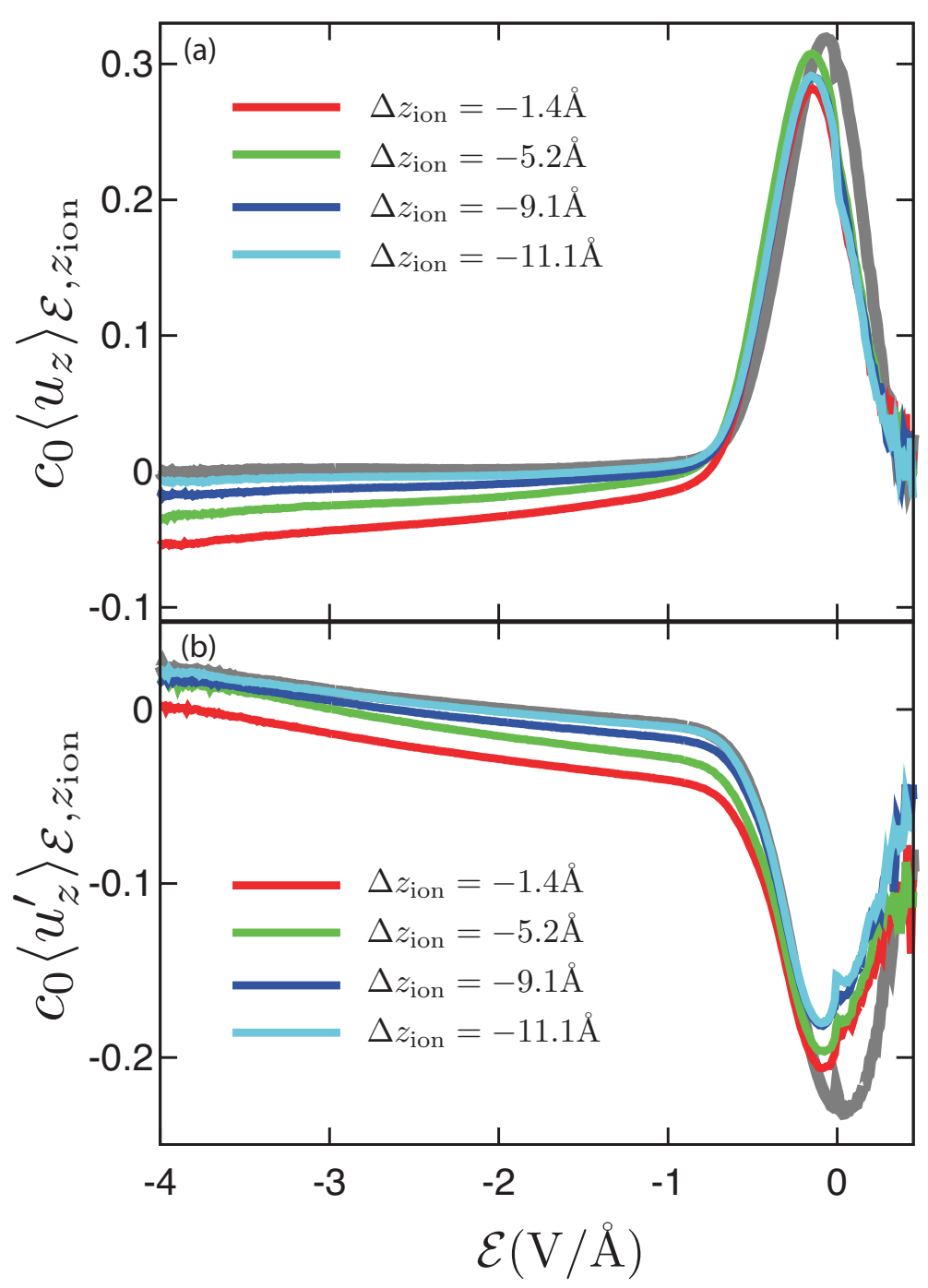

FIG. 4: Dependence of a liquid slab's orientational bias on the depth of a dissolved cation. Average projections of $\mathrm{OH}(\mathrm{a})$ and $\mathrm{OD}(\mathrm{b})$ bond vectors onto the surface normal are shown as functions of electric field for a series of systems whose $\mathrm{Na}^{+}$cation is restrained to different ranges of $\Delta z_{\text {cation }}=$ $z_{\text {cation }}-z_{\text {Gibbs }}$. In each case $\sqrt{\left\langle\left(z_{\text {cation }}-\left\langle z_{\text {cation }}\right\rangle\right)^{2}\right\rangle}=0.5 \AA$. Results for the neat liquid (gray curves) are included for comparison. 


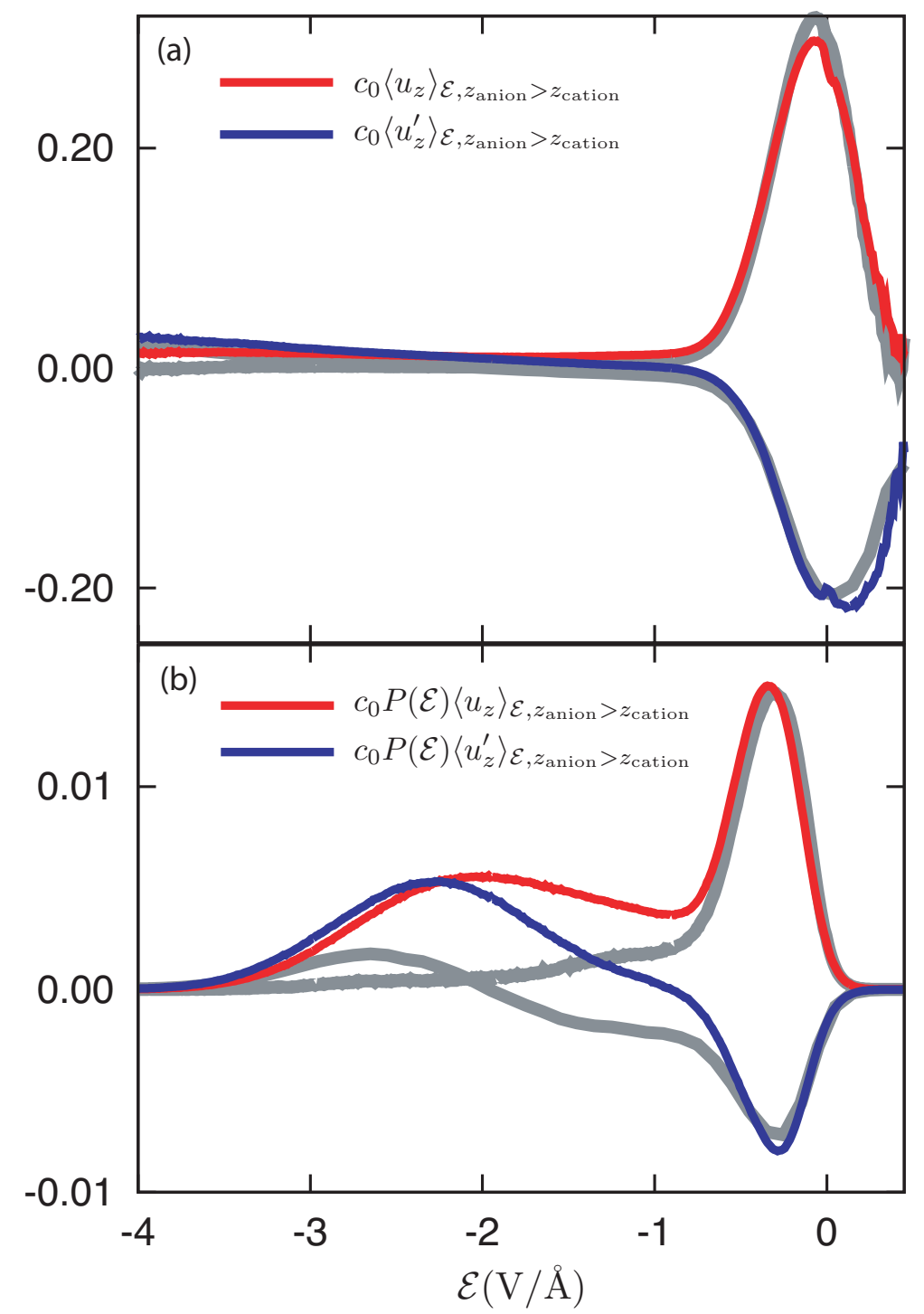

FIG. 5: Estimate of orientational averages governing SFG response for a stratified ion pair at infinite dilution. The plotted data were obtained by averaging calculations for a single dissolved $\mathrm{I}^{-}$anion, held at depth $\left\langle\Delta z_{\text {anion }}\right\rangle=-1.2 \AA$, with calculations for a single dissolved $\mathrm{Na}^{+}$cation, held at lower $\operatorname{depth}\left\langle\Delta z_{\text {cation }}\right\rangle=-7.1 \AA$. Depth of both ions was allowed to fluctuate by $\sqrt{\left\langle\left(z_{\text {ion }}-\left\langle z_{\text {ion }}\right\rangle\right)^{2}\right\rangle}=$ 0.5A. Panel (a) shows $c_{0}\left\langle u_{z}\right\rangle_{\mathcal{E}, z_{\text {ion }}}$ and $c_{0}\left\langle u_{z}^{\prime}\right\rangle_{\mathcal{E}, z_{\text {ion }}}$. In (b) these average projections have been weighted by the electric field distribution in order to highlight differences with results for the neat liquid (gray). 


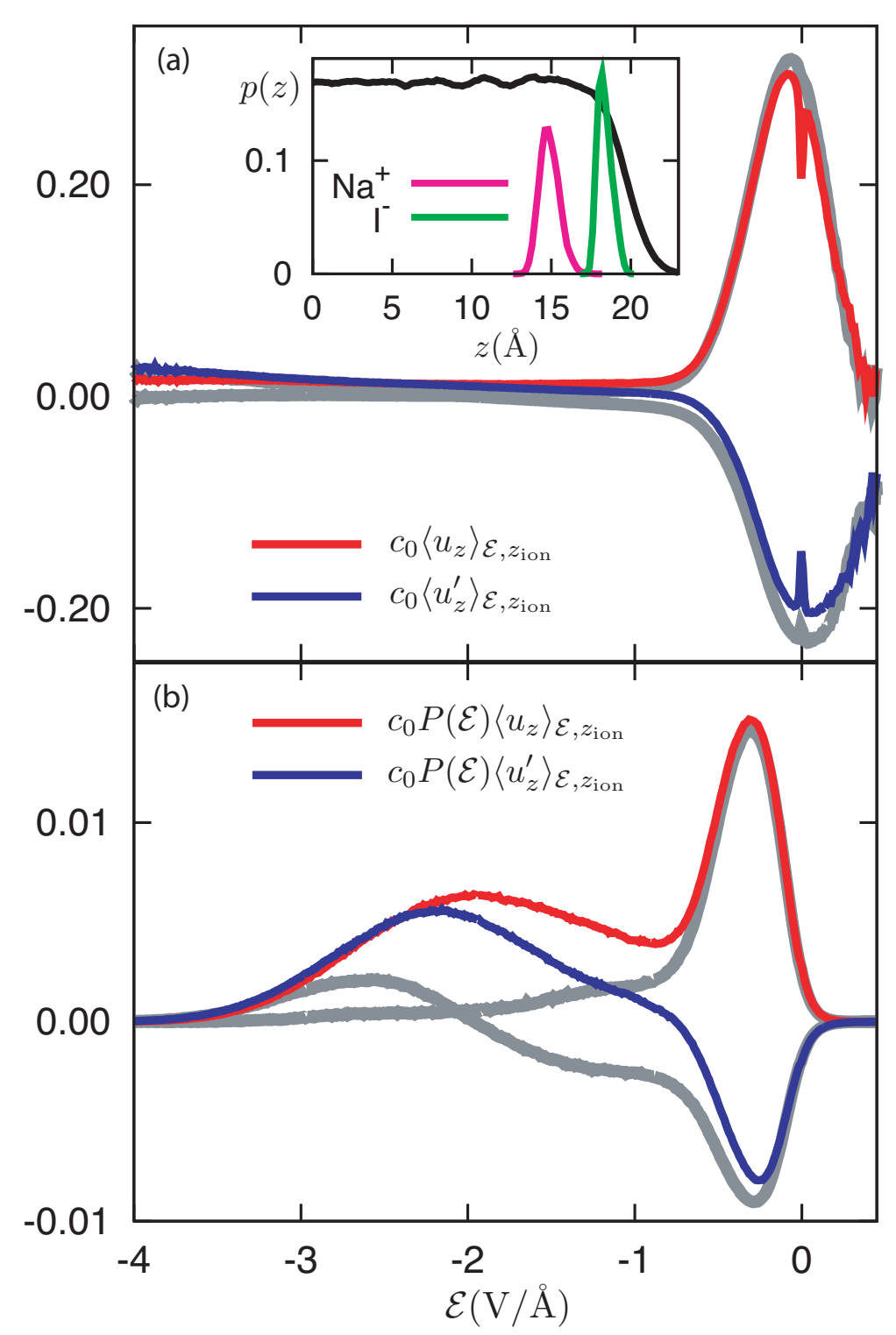

FIG. 6: Average projections of $\mathrm{OH}$ and $\mathrm{OD}$ bond vectors onto the surface normal for a liquid slab containing a pair of ions held at different depths, but in close proximity, near the interface. Subject to the bias potentials described in the text, the $\mathrm{I}^{-}$anion fluctuates about a mean depth $\left\langle\Delta z_{\text {anion }}\right\rangle=-1.5 \AA$ by an amount $\sqrt{\left\langle\left(z_{\text {anion }}-\left\langle z_{\text {anion }}\right\rangle\right)^{2}\right\rangle}=0.5 \AA$. The $\mathrm{Na}^{+}$cation resides at a mean depth $\left\langle\Delta z_{\text {cation }}\right\rangle=-5 \AA$ and undergoes vertical fluctuations of size $\sqrt{\left\langle\left(z_{\text {cation }}-\left\langle z_{\text {cation }}\right\rangle\right)^{2}\right\rangle}=$

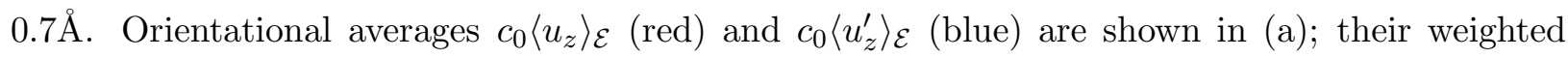
counterparts $c_{0} P(\mathcal{E})\left\langle u_{z}\right\rangle_{\mathcal{E}}$ and $c_{0} P(\mathcal{E})\left\langle u_{z}^{\prime}\right\rangle_{\mathcal{E}}$ are shown in (b). Results for the neat liquid (gray) are included for comparison. The inset in (a) depicts the solvent density profile (scaled by an arbitrary constant) as well as distributions of $\operatorname{depth} p(z)$ for the two ions. 


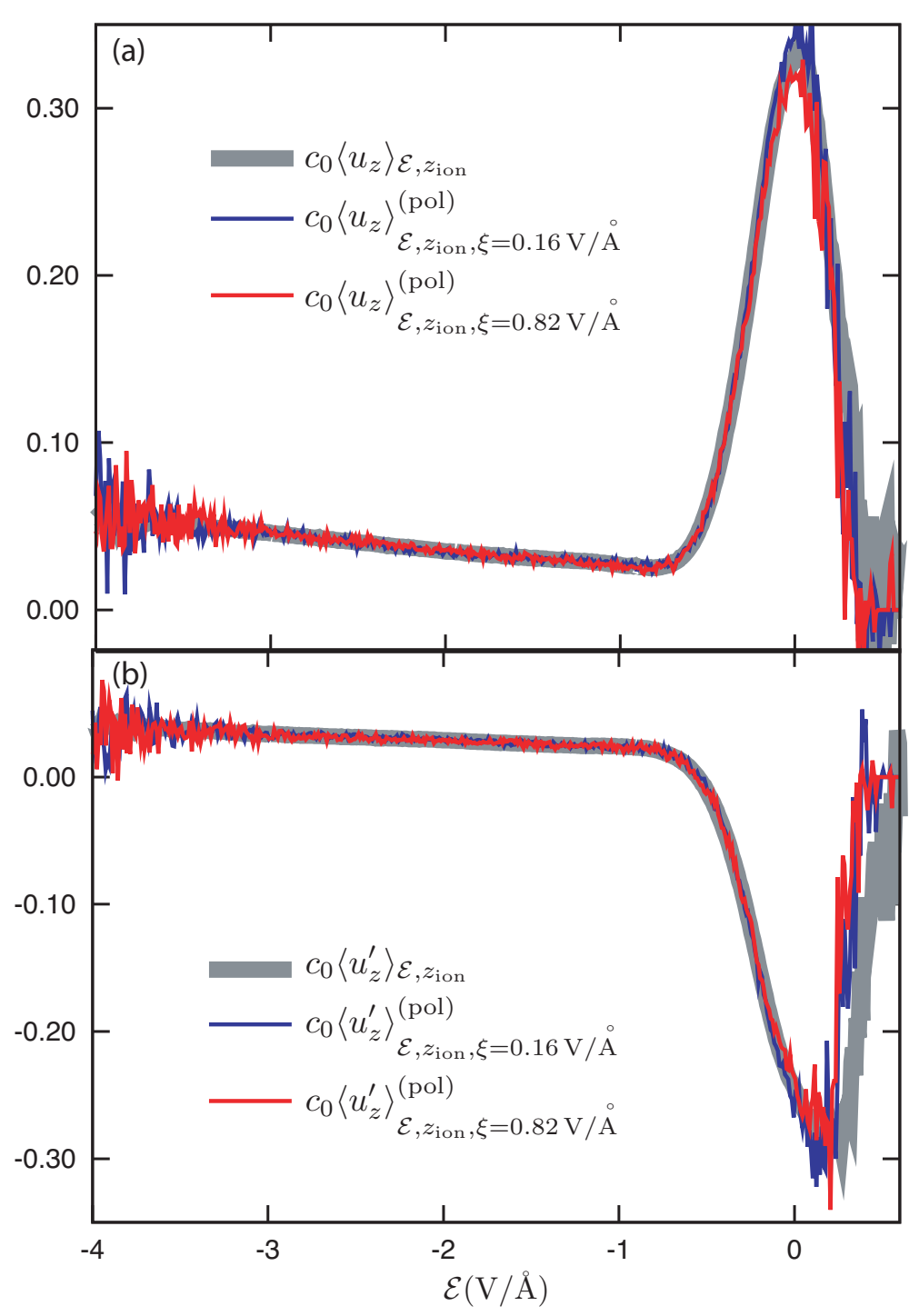

FIG. 7: Influence of dipole induction on orientational averages when a single $\mathrm{I}^{-}$anion is held fixed at depth $\left\langle\Delta z_{\text {anion }}\right\rangle=-1.3 \AA$ with vertical fluctuations of size $\sqrt{\left\langle\left(z_{\text {anion }}-\left\langle z_{\text {anion }}\right\rangle\right)^{2}\right\rangle}=0.5 \AA$. Red and blue curves show the conditional averages $\left\langle u_{z}\right\rangle_{\mathcal{E}, z_{\text {ion }}}^{(0)}$ and $\left\langle u_{z}^{\prime}\right\rangle_{\mathcal{E}, z_{\text {ion }}}^{(0)}$ for two extreme values of the electric field $\xi$ acting on the ion (which determines dipole induction). For comparison, we reproduce the results from Fig. 1 for a non-polarizable iodide anion (gray curves). The thermal distribution of $\xi$ in these circumstances includes $93 \%$ of its weight between $\xi=0.16 \mathrm{~V} / \AA$ (blue curves) and $\xi=0.82 \mathrm{~V} / \AA$ (red curves). 


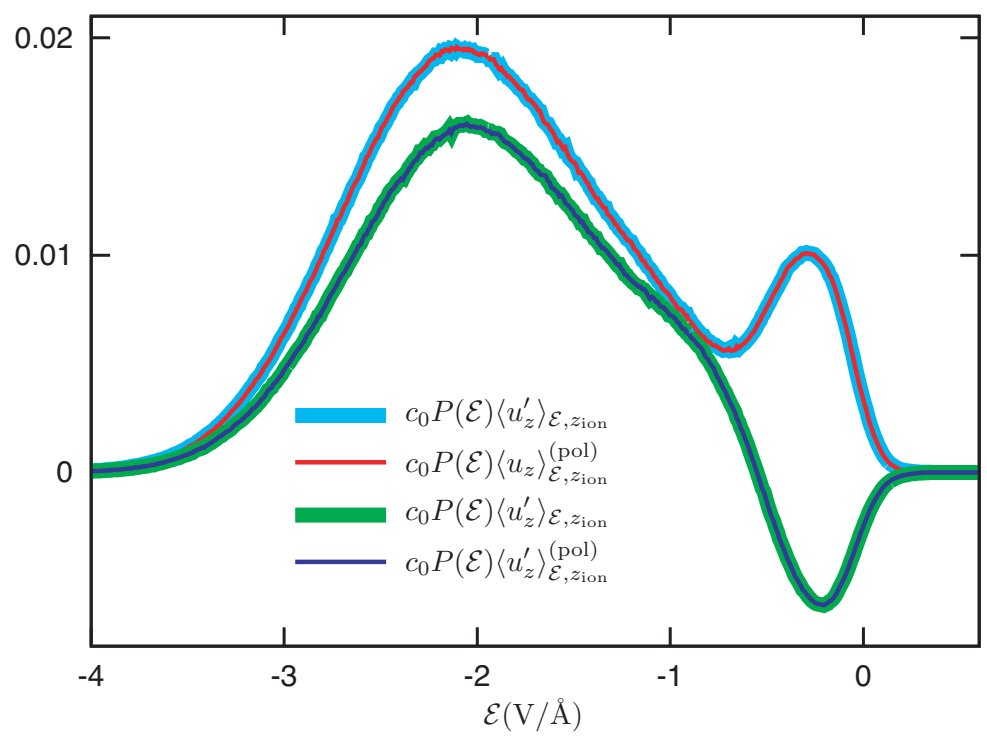

FIG. 8: Weighted orientational averages, $P(\mathcal{E})\left\langle u_{z}\right\rangle_{\mathcal{E}, z_{\text {ion }}}^{(\mathrm{pol})}$ and $P(\mathcal{E})\left\langle u_{z}^{\prime}\right\rangle_{\mathcal{E}, z_{\text {ion }}}^{(\mathrm{pol})}$, underlying SFG response for a polarizable iodide anion held at $\left\langle\Delta z_{\text {anion }}\right\rangle=-1.3$ with $\sqrt{\left\langle\left(z_{\text {anion }}-\left\langle z_{\text {anion }}\right\rangle\right)^{2}\right\rangle}=0.5 \AA$. Plotted results (blue and red curves) were calculated from Eq. 8 with polarizability $\alpha=6.92 \AA^{3}[24]$. For comparison we reproduce the results from Fig. 1 for a non-polarizable iodide anion (cyan and green curves). 